Cochrane Database of Systematic Reviews

\title{
Pressure redistributing static chairs for preventing pressure ulcers
} (Protocol)

Stephens M, Bartley C, Dumville JC, Cammiss CJ

Stephens M, Bartley C, Dumville JC, Cammiss CJ.

Pressure redistributing static chairs for preventing pressure ulcers (Protocol).

Cochrane Database of Systematic Reviews 2020, Issue 6. Art. No.: CD013644.

DOI: 10.1002/14651858.CD013644.

www.cochranelibrary.com 
TABLE OF CONTENTS

HEADER 1

ABSTRACT

BACKGROUND

OBJECTIVES

METHODS

ACKNOWLEDGEMENTS

REFERENCES

APPENDICES

HISTORY

CONTRIBUTIONS OF AUTHORS

DECLARATIONS OF INTEREST

SOURCES OF SUPPORT 
[Intervention Protocol]

\section{Pressure redistributing static chairs for preventing pressure ulcers}

Melanie Stephens ${ }^{1}$, Carol Bartley², Jo C Dumville ${ }^{3}$, Christopher J Cammiss ${ }^{4}$

${ }^{1}$ School of Health and Society, University of Salford, Salford, UK. ${ }^{2}$ Rehab For Independence, Chorley, UK. ${ }^{3}$ Division of Nursing, Midwifery and Social Work, School of Health Sciences, Faculty of Biology, Medicine and Health, University of Manchester, Manchester Academic Health Science Centre, Manchester, UK. ${ }^{4}$ Disabled Living, Salford, UK

Contact address: Melanie Stephens, stephens.melanie@outlook.com, m.stephens@salford.ac.uk.

Editorial group: Cochrane Wounds Group.

Publication status and date: New, published in Issue 6, 2020.

Citation: Stephens M, Bartley C, Dumville JC, Cammiss CJ. Pressure redistributing static chairs for preventing pressure ulcers (Protocol). Cochrane Database of Systematic Reviews 2020, Issue 6. Art. No.: CD013644. DOI: 10.1002/14651858.CD013644.

Copyright @ 2020 The Cochrane Collaboration. Published by John Wiley \& Sons, Ltd.

\section{A B S T R A C T}

\section{Objectives}

This is a protocol for a Cochrane Review (intervention). The objectives are as follows:

To assess the effects of pressure redistributing static chairs on the prevention of pressure ulcers in health, rehabilitation, social care settings, and places of residence in which people may spend their day. 


\section{B A C K G R O U N D}

\section{Description of the condition}

Pressure ulcers are a global issue that have serious consequences for those people affected and for health systems more widely. A pressure ulcer is defined as "localized injury to the skin and/or underlying tissue usually over a bony prominence, as a result of pressure, or pressure in combination with shear" (NPIAP 2016). Historically, the term pressure ulcer has changed from bedsore, decubitus ulcer, and pressure sore, to pressure ulcer and pressure injury. In Europe the term pressure ulcer has been adopted; whilst in North America, Asia, and Australasia the term pressure injury is preferred.

Pressure ulcer severity is staged or categorised from 1 to 4 (EPUAP 2014; NPIAP 2016; WHO 2018). The higher the category the more significant the pressure ulcer and damage to the skin and underlying tissues, with stage/category 1 indicating superficial damage with no open wound and stage/category 4 indicating significant damage through the layers of the skin, soft tissue, and muscle, often exposing bone.

Prevalence estimates for pressure ulceration vary according to the population being assessed, the data collection methods used, and decisions about whether or not category I pressure ulcers should be included (since there is no open wound at this stage but evidence of possible tissue damage). Current estimates of the prevalence of pressure ulcers from a systematic review of the data vary from $0.056 \%$ to 23\% (Cullum 2016). The point prevalence of pressure ulceration in the total adult population was estimated in literature produced between 1997 to 2009 using a cross-sectional survey undertaken in Leeds, UK. Of the total adult population of 751,485 , the point prevalence of pressure ulceration per 1000 was 0.31 (Cullum 2016). UK pressure ulcer prevalence estimates specifically for community settings have reported rates of 0.77 per 1000 adults in a UK urban area (Stevenson 2013).

The financial implications of pressure ulceration to health services is large, and estimated across countries as from EUR 12.58 million to EUR 240.94 million for prevention, to EUR 121.44 million to EUR 2.59 billion for treatment (Demarré 2015). A recent retrospective cohort analysis found that UK National Health Service (NHS) community costs of pressure ulcer treatment ranged from GBP 1400 for a category 1 ulcer to over GBP 8500 for the other categories (Guest 2018).

Being seated with no or limited movement increases the risk of pressure ulceration as soft tissues are compressed between two surfaces, the seat and the bones of the pelvis, for long periods (Krouskop 1983; Schubert 1994). This starves the area of oxygen and nutrients and, if pressure is unrelieved, skin begins to break down (Kosiak 1959). The risk of ulceration and rate of development can vary, as high pressures over a short period of time can be equally as destructive as low pressures over a longer period (Gefen 2008). Damage from shear occurs when layers of the skin are laterally shifted in relation to each other because of limited movement across a surface. Immobilisation or reduced mobility are risk factors for pressure ulceration, as is reduced bodily sensation; other suggested risk factors include unmanaged incontinence (resulting in constantly moist skin), poor circulation, and poor nutrition (Bhattacharya 2015; Bartley 2017). A pressure ulcer can begin with inflamed, sore, painful skin. However, if a person has reduced bodily sensation, the initial feelings of soreness are not felt and an open wound could be the first signs of skin damage.

There are approximately $131,800,000$ wheelchair users worldwide who are not ambulant and are at increased risk for the development of a pressure ulcer (Wheelchair Foundation 2019). Also at risk of ulceration are those who have limited mobility and sit in static chairs for extended periods of time, for example elderly and frail populations: the number of people in this at-risk group is difficult to estimate but likely to be very large. For some patients, sitting can be viewed as a therapeutic intervention and an important part of a patient's recovery process; but the risk of ulceration also has to be mitigated. Interventions for ulcer prevention in those at risk from prolonged sitting include the use of specialist cushions and surfaces (such as reactive and active, powered and non-powered devices (NPIAP 2019)), especially in wheelchair users (Bartley 2017; Stephens 2017). There is also increased interest in the effects of different pressure-redistributing static chairs on pressure ulcer development in at-risk people.

\section{Description of the intervention}

Pressure redistributing static chairs as devices for those who remain seated for extended periods of time due to short- or longterm mobility issues, or both, are used in many settings, including hospitals, care homes, and people's own homes. They are an essential part of the 24-hour postural management programme for this population, and are considered a key assistive device for promoting independence and function (Bartley 2017; Harrand 2016; Stephens 2017). There are many different types of static chair available for use in a healthcare context, with various levels of pressure redistributing technology: ranging from standard hospital chairs and chairs used in residential settings with no redistribution function, to those that include foam, gel, air, or water with the aim of reducing pressure on soft tissue. These can include:

- static arm chairs, known as a high-back chair, which is a high-seat chair with an upright seating position. It comes with additional lumbar support, optional wings (side pieces projecting from the back of the chair) and arms that can be upholstered in antimicrobial vinyls and fabric upholstery options and a removable seat cushion;

- recliner chair: a high-back chair, which is a high-seat chair with a sculpted or waterfall (sloping) back that reclines when the occupant lowers the chair's back and raises its front. It has a backrest that can be tilted back, and often a footrest that may be extended by means of a lever on the side of the chair, or may extend automatically when the back is reclined. It can also have lift and tilt actions;

- riser: a recliner chair (single motor/action) is a high-back chair, which is a high-seat chair with a sculpted or a waterfall back that provides a two-way, tilt-in-space action that gives high leg elevation as the seat to back angle remains constant. The tilt in space can be controlled manually or through a handset. It can also have lift and tilt actions;

- riser: a recliner chair (dual motor/action) is a high-back chair, which is a high-seat chair with a sculpted or waterfall back, utilising two motors, operated by a handset to allow independent leg rest and backrest operation. The backrest can be reclined virtually flat as the backrest opens away from the seat. It can also have lift and tilt actions; 
- hybrid specialist seating: manual or dynamic seating systems that provide positioning and postural management for people who are at risk of developing an asymmetrical body shape, unable to maintain a midline position and require additional postural support. The seating can be adjusted for the correct seat height, seat depth, seat width, arm height, and back height combined with headrest angle adjustment, back angle recline, tilt-in-space, and leg rest elevation. Pressure redistributing seat cushions can be integrated into the chair;

- bespoke custom-configured seating to accommodate more complex postures or skin integrity needs that cannot be met with off-the-shelf seating solutions;

- bespoke custom-moulded seating to accommodate the fixed asymmetrical posture of people who require significant postural support.

The number of different static chairs with pressure redistributing elements has increased exponentially since the publication of the 2008 Tissue Viability Seating Guidelines, despite no standardised terminology, for example the UK NHS Supply Chain currently lists 307 static chairs ranging in cost from GBP 270 to GBP 2500 (NHS Supply Chain 2019), and this excludes bespoke equipment e.g. moulded seat inserts fitted to the person's requirements.

\section{How the intervention might work}

To reduce pressure ulcer risk when seated, static chairs aim to reduce seating pressure around the ischial tuberosities (bony parts of the buttocks), greater trochanters (widest part of the thigh), and sacral (area at the top of the buttock crease) region (Reddy 2006). However, attention should also be paid to other bony prominences, such as shoulder blades, spinal processes, back of head, elbows, and heels (Stephens 2017). The aim is that the support provided by the chair minimises constant pressure allowing good blood flow to the soft tissues, maintaining the metabolic needs of local cells, and reducing the risk of ulceration (Jan 2010). Several approaches are used in the development of static chairs to redistribute pressure and prevent shear or friction forces. Features such as seat cushion design; tilt-in-space or recline function, or both; and use of elevated leg rests are all used in an attempt to address these concepts (Stinson 2003; Michael 2007; RESNA 2009; Jan 2010; Jan 2013; NICE 2014). However, much of the testing of these features has been undertaken in wheelchair design and not static chair design.

\section{Why it is important to do this review}

Currently there is no systematic review focused on the evidence for different types of static chairs in pressure ulcer prevention and there is a potential over-reliance on marketing material to inform decision-making in this area. Given the widespread use of static chairs with pressure redistributing technology in health and social care settings, it is important to systematically review current evidence for their effects. Whilst previous reviews have focused on cushions and wheelchairs, this Cochrane Review will consider the different types of static chairs and related seat moulds used in health and social care contexts, the evidence for the effectiveness of these, and any outstanding uncertainty that may shape future research and use of healthcare finances.

\section{OB JECTIVES}

To assess the effects of pressure redistributing static chairs on the prevention of pressure ulcers in health, rehabilitation, social care settings, and places of residence in which people may spend their day.

\section{METHODS}

\section{Criteria for considering studies for this review}

\section{Types of studies}

We will include published and unpublished randomised controlled trials (RCTs), including cluster-RCTs, irrespective of language of report. We will exclude quasi-randomised studies.

\section{Types of participants}

We will include studies in adults who remain seated for extended periods of time, undertaken in any care setting: this might include health, rehabilitation, social care settings, and places of residence in which people may spend their day. We will include people at risk of developing new pressure ulcers, which may include those with existing pressure ulcers at baseline when pressure ulcer incidence was measured in the study.

\section{Types of interventions}

We will include any type of pressure redistributing static chair. This may include, but is not limited to: static arm chairs, riser recline chairs, tilt-in-space chairs, recline chairs, and hybrid chairs. We are not considering comparisons of static chairs with wheelchairs, or of different wheelchairs with each other in this review as wheelchairs are mobility aids, nor is the focus of this review the effects of different types of pressure redistributing cushions, as these are included in a Cochrane Review focused on support surfaces (McInnes 2015). We will include trials where the only systematic difference between trial arms is the use of a specific static chair type.

We anticipate that comparisons might include but not be limited to:

- pressure redistributing chairs compared with a standard chair (used in a ward or residential setting) which may include:

* pressure redistributing static chair compared with standard chair;

* pressure redistributing recline chair compared with standard chair;

* pressure redistributing riser recline chair compared with standard chair;

* pressure redistributing tilt in space chair compared with standard chair;

* pressure redistributing hybrid chair compared with standard chair;

- comparison of different types of pressure redistributing chairs which may include, but not be limited to, any combination of the chair types noted above.

Classification of intervention type will be based on information presented in the paper. Standard chairs used in a ward or residential setting can have a high or low back, arm rests, front cross bars (horizontal support element joining the legs), removable seat cushions, custom-moulded seat inserts, with or without wings and side infill. We will use study authors' definition of a standard chair unless it is clear this is very different to this general definition. 


\section{Types of outcome measures}

We list primary and secondary outcomes below. If a study is otherwise eligible (i.e. correct study design, population, and intervention/comparator) but does not report a listed outcome, then we will contact the study authors where possible to establish whether an outcome of interest here was measured but not reported.

We will report outcome measures at the latest time point available (assumed to be length of follow-up if not specified) and the time point specified in the methods as being of primary interest (if this is different from the latest time point available). For all outcomes we will class assessment of outcome measures as:

- $<1$ week to 8 weeks as short term;

- $>8$ weeks to 26 weeks as medium term; and

- $>26$ weeks as long term.

\section{Primary outcomes}

\section{Pressure ulcer incidence}

- Proportion of participants developing a new pressure ulcer. These data will include all incidence ulcers regardless of grade/ category (1 to 4);

- time-to-pressure ulcer development, correctly analysed using survival, time-to-event approaches. Mean time to event data will not be extracted unless it is clear that all participants in the study developed an ulcer (a scenario which we anticipate being highly unlikely).

\section{Secondary outcomes}

- Participant health-related quality of life/health status: (measured using a standardised generic questionnaire such as EQ-5D (Herdman 2011), Short Form (SF)-36, (Ware 1992), SF-12 (Ware 1996), or SF-6 (Brazier 2002)). We will not include ad hoc measures of quality of life that are unlikely to be validated and would not be common to multiple trials;

- participant comfort: we are not aware of any validated scale for patient comfort so this measure will be as reported by study authors;

- cost effectiveness: within-trial cost-effectiveness analysis comparing mean differences in effects with mean cost differences between the two study arms: data extracted will be incremental mean cost per incremental gain in benefit (incremental cost-effectiveness ratios) or other combined measures of cost and effects.

- All reported adverse events. We will include data where study authors have specified a clear method for collecting adverse event data. Where available, we will extract data on all serious and all non-serious adverse events as an outcome. We will record where it is clear that events are reported at the participant level or whether multiple events per person were reported, and then if appropriate adjustments were made for data clustering. We will consider the assessment of any event in general defined as adverse by participants, health professionals, or both.

\section{Search methods for identification of studies}

\section{Electronic searches}

We will search the following databases to retrieve reports of relevant trials:

- the Cochrane Wounds Specialised Register (to present);

- the Cochrane Central Register of Controlled Trials (CENTRAL) in the Cochrane Library (to latest issue);

- Ovid MEDLINE (from 1946 to present);

- Ovid MEDLINE (In-Process \& Other Non-Indexed Citations);

- Ovid Embase (from 1974 to present);

- EBSCO CINAHL Plus (from 1937 to present).

We have devised a draft search strategy for CENTRAL which is displayed in Appendix 1. We will adapt this strategy to search the Cochrane Wounds Specialised Register, Ovid MEDLINE, Ovid Embase and EBSCO CINAHL Plus. We will combine the Ovid MEDLINE search with the Cochrane Highly Sensitive Search Strategy for identifying randomised trials in MEDLINE: sensitivityand precision-maximising version (2008 revision) (Lefebvre 2019). We will combine the Embase search with the Ovid Embase filter terms developed by the UK Cochrane Centre (Lefebvre 2019). We will combine the CINAHL Plus search with the trial filter developed by Glanville 2019. There will be no restrictions of the searches by language, date of publication or study setting.

We will also search the following clinical trials registries for ongoing studies:

- ClinicalTrials.gov (www.clinicaltrials.gov);

- WHO International Clinical Trials Registry Platform (www.who.int/trialsearch).

\section{Searching other resources}

We aim to identify other potentially eligible trials or ancillary publications by searching the reference lists of retrieved included trials, as well as relevant systematic reviews, meta-analyses, and health-technology assessment reports.

When necessary, we will contact experts in wound care and medical devices to enquire about unpublished, ongoing, and recently published trials.

We will not perform a separate search for adverse effects of chair use. We will consider adverse effects described in included studies only.

\section{Data collection and analysis}

\section{Selection of studies}

Two review authors will independently assess the titles and abstracts of the citations retrieved by the searches for relevance. After this initial assessment, we will obtain full-text copies of all studies considered to be potentially relevant. Two review authors will independently check the full papers for eligibility; disagreements will be resolved by discussion and, where required, the input of a third review author. Where required and possible, we will contact study authors where the eligibility of a study is unclear. We will record all reasons for exclusion of studies for which we 
had obtained full copies. We will complete a PRISMA flowchart to summarise this process (Liberati 2009).

Where studies have been reported in multiple publications/reports, we will obtain all publications. Whilst we will include each study only once in the review, we will extract data from all reports to ensure we obtain maximal relevant data.

\section{Data extraction and management}

We will extract and summarise details of the eligible studies using a data extraction sheet. Two review authors will extract data independently and will resolve disagreements by discussion, and will consult a third review author where required. Where data are missing from reports, we will attempt to contact the study authors to obtain these. Where we have included a study with more than two intervention arms, we will only extract data from intervention and control groups that meet the eligibility criteria.

We will extract the following data where possible by treatment group for the pre-specified interventions and outcomes in this review. We will collect outcome data for relevant time points as described in the Types of outcome measures section:

- country of origin;

- type of setting;

- trial design and unit of randomisation: was randomisation undertaken the participant level or at the level of a ward, care home, or other location;

- unit of analysis: how were clustered data addressed in the analysis;

- care setting;

- number of participants randomised to each trial arm;

- eligibility criteria and key baseline participant data (participant age, gender, and skin status where recorded);

- details of treatment regimen received by each group;

- duration of treatment;

- details of any co-interventions;

- primary and secondary outcome(s) (with definitions);

- outcome data for primary and secondary outcomes (by group);

- duration of follow-up;

- number of withdrawals (by group);

- publication status of study; and

- source of funding for trial.

\section{Assessment of risk of bias in included studies}

Two review authors will independently assess the risk of bias in included studies by using the Cochrane 'Risk of bias' tool (Higgins 2011a). This tool addresses six specific domains: sequence generation, allocation concealment, blinding, incomplete data, selective outcome reporting, and other issues. In this review we will record issues with unit of analysis, for example where a cluster-randomised trial has been undertaken but analysed at the individual level in the study report (Appendix 1). We will assess blinding and completeness of outcome data for each of the review outcomes separately. We note that, since pressure ulcer incidence is a subjective outcome, it can be at high risk of measurement bias when outcome assessment is not blinded. We will consider studies without blinded outcome assessment of ulcer incidence at high risk of detection bias. It will be unlikely that studies are able to blind health professionals or patients to treatment received, meaning that most or all studies will be regarded as being at high risk of performance bias. However, where studies have documented their processes to minimise differences in performance, for example with protocol-guided delivery of co-interventions, we may make a judgement of unclear or low risk of bias. We will present our assessment of risk of bias using two 'Risk of bias' summary figures: one will be a summary of bias for each item across all studies, and the second will show a cross-tabulation of each trial by all 'Risk of bias' items. We will class studies with an assessment of low risk of bias for selection bias (both domains of sequence generation and allocation concealment), detection bias, and attrition bias, and no assessment of high risk of bias in any other domain, to be at overall low risk of bias (for specified outcome). Studies with a high risk of bias assessment in any domains will be classed as being at high risk of bias.

For cluster-randomised trials, we will also consider the risk of bias in terms of: recruitment bias, baseline imbalance, loss of clusters, incorrect analysis, and comparability with individually randomised trials (Higgins 2011b; Appendix 2).

\section{Measures of treatment effect}

For dichotomous outcomes we will calculate the risk ratio (RR) with $95 \%$ confidence intervals $(\mathrm{Cl})$. For continuously distributed outcome data we will use the mean difference (MD) with 95\% Cls, if all trials use the same or similar assessment scale. If trials use different assessment scales, we will use the standardised mean difference (SMD) with $95 \%$ Cls. We will only consider mean or median time to healing without survival analysis as a valid outcome if reports specify that all wounds healed (i.e. if the trial authors regarded time to healing as a continuous measure as there is no censoring). We will report time-to-event data (e.g. time-to-pressure ulceration), as hazard ratios (HR) where possible in accordance with the methods described in the Cochrane Handbook for Systematic Reviews of Interventions (Deeks 2011). If studies reporting time-toevent data do not report an HR, then, where feasible, we plan to estimate this using other reported outcomes, such as the numbers of events, through the application of available statistical methods (Parmar 1998).

\section{Unit of analysis issues}

Some studies randomise by participant, but analyse outcomes by wound. Where this occurs and the numbers of participants and wounds are equal (i.e. one wound per participant), we will treat the participant as the unit of analysis. There may be instances of clustered data, where a proportion of trial participants have outcome data collected and reported on multiple wounds. Since not all participants will have multiple wounds this is not a cluster trial per se, but rather a trial that incorrectly includes a mixture of individual and clustered data. We will note such trials and will record the issue in the 'Risk of bias' assessment. Data will be extracted and presented, but will not be the subject of any further analyses.

We will only incorporate correctly designed and analysed full cluster trials into meta-analyses. Where a cluster-randomised trial has been conducted but incorrectly analysed, we will record this in the 'Risk of bias' assessment. If it is possible, we will approximate the correct analyses with guidance from the Cochrane Handbook for Systematic Reviews of Interventions (Higgins 2011b), using information on: 
- the number of clusters randomised to each intervention, or the mean size of each cluster;

- outcome data ignoring cluster design for the total number of individuals; and

- an estimate of the intracluster correlation coefficient (ICC).

If we cannot analyse the study data correctly, we will extract and present the data without further analysis.

We will ensure there are no unit of analysis issues with double counting of controls when using studies with multiple intervention arms.

Where repeated observations are recorded on the same participant (e.g. comfort scales over time), we will define time points and analyse accordingly. Where multiple recordings are available within these time points we will incorporate all available data for an overall mean where possible.

\section{Dealing with missing data}

It is common for there to be to data missing from trial reports. Excluding participants post-randomisation from the analysis, or ignoring those participants who are lost to follow-up compromises the randomisation, and potentially introduces bias into the trial. Where there are missing data that we think should be included in the analyses, we will contact the relevant study authors to request these data.

Where data remain missing for the 'proportion of participants developing a new pressure ulcer' outcome, for analysis we will assume that if randomised participants were not included in an analysis, they did not have an incident ulcer (i.e. they would be considered in the denominator but not the numerator). We will explore this in a sensitivity analysis where the impact of no imputation (complete case analysis) is considered (see Sensitivity analysis).

In a time-to-pressure ulceration analysis using survival analysis methods, drop-outs should be accounted for as censored data and we will not take any action regarding missing data.

For all secondary outcomes we will present available data from the study reports/study authors and do not plan to impute missing data. Where measures of variance are missing, we will calculate these where possible. If calculation is not possible, we will contact the study authors for further information. Where these measures of variance are not available, we will exclude the study from any relevant meta-analyses that we conduct.

\section{Assessment of heterogeneity}

Assessment of heterogeneity can be a complex, multi-faceted process. Firstly, we will consider clinical and methodological heterogeneity, that is the degree to which the included studies vary in terms of participant, intervention, outcome, and characteristics such as length of follow-up. We will supplement this assessment of clinical and methodological heterogeneity by information regarding statistical heterogeneity - assessed using the $\mathrm{Chi}^{2}$ test (we will consider a significance level of $P<0.10$ to indicate statistically significant heterogeneity) in conjunction with the 12 measure (Higgins 2003). The 12 statistic examines the percentage of total variation across RCTs that is due to heterogeneity rather than chance (Higgins 2003). In general, 12 values of $25 \%$ or less may mean a low level of heterogeneity (Higgins 2003), and values of more than $75 \%$ indicate very high heterogeneity (Deeks 2011). Where there is evidence of high heterogeneity we will attempt to explore this further (see Data synthesis).

\section{Assessment of reporting biases}

Reporting biases arise when the dissemination of research findings is influenced by the nature and direction of results. Publication bias is one of a number of possible causes of 'small study effects', that is, a tendency for estimates of the intervention effect to be more beneficial in smaller RCTs. Funnel plots allow a visual assessment of whether small study effects may be present in a meta-analysis. A funnel plot is a simple scatter plot of the intervention effect estimates from individual RCTs against some measure of each trial's size or precision (Sterne 2011). We plan to present funnel plots for meta-analyses comprising 10 RCTs or more using Review Manager 5 (RevMan 5) (Review Manager 2014).

\section{Data synthesis}

We will combine details of included studies in a narrative review according to type of comparator, possibly by location/type of wound, and then by outcomes by time period. We will consider clinical and methodological heterogeneity and will undertake pooling when studies appear appropriately similar in terms of wound type, intervention type, duration of follow-up, and outcome type.

We cannot pre specify the amount of clinical, methodological, and statistical heterogeneity in the included studies, but it may be extensive. Thus, we anticipate using a random-effects approach for meta-analysis. Conducting meta-analysis with a fixed-effect model in the presence of even minor heterogeneity may provide overly narrow Cls. We will only use a fixed-effect approach when clinical and methodological heterogeneity is assessed to be minimal, and the assumption that a single underlying treatment effect is being estimated holds. We will use $\mathrm{Chi}^{2}$ and $\mathrm{I}^{2}$ tests to quantify heterogeneity but we will not used these to guide choice of model for meta-analysis. We will exercise caution when meta-analysed data are at risk of small study effects because a random-effects model may be unsuitable. In this case, or where there are other reasons to question the selection of a fixed-effect or random-effects model, we will assess the impact of the approach using sensitivity analyses to compare results from alternate models. We will report any evidence that suggests that the use of a particular model might not be robust. We may meta-analyse even when there is thought to be extensive heterogeneity. We will attempt to explore the causes behind this using meta-regression, if possible (Thompson 1999).

We will present data using forest plots where possible. For dichotomous outcomes we will present the summary estimate as a risk ratio (RR) with $95 \% \mathrm{Cl}$. Where continuous outcomes are measured in the same way across studies, we plan to present a pooled mean difference (MD) with $95 \% \mathrm{Cl}$; we plan to pool standardised mean difference (SMD) estimates where studies measure the same outcome using different methods. For time-toevent data, we plan to plot (and, if appropriate, pool) estimates of HRs and $95 \% \mathrm{Cls}$ as presented in the study reports using the generic inverse variance method in RevMan 5 (Review Manager 2014). Where time-to-pressure ulcer development is analysed as a continuous measure but it is not clear if all participants develop a pressure ulcer (an unlikely scenario), we will document use of the 
outcome in the study but we will not summarise data or use them in any meta-analysis.

We will obtain pooled estimates of treatment effect using RevMan 5 (Review Manager 2014).

\section{'Summary of findings' tables and assessment of the certainty of evidence}

We will present the main results of the review in 'Summary of findings' tables, at this stage we anticipate including data from all comparisons in 'Summary of findings' tables. These tables present key information concerning the certainty of the evidence, the magnitude of the effects of the interventions examined, and the sum of the available data for the main outcomes (Schünemann 2011a). The 'Summary of findings' tables also include an overall grading of the evidence related to each of the main outcomes using the GRADE approach. The GRADE approach defines the certainty of a body of evidence as the extent to which one can be confident that an estimate of effect or association is close to the true quantity of specific interest. The certainty of a body of evidence involves consideration of within-trial risk of bias (methodological quality), directness of evidence, heterogeneity, precision of effect estimates, and risk of publication bias (Schünemann 2011b). We plan to present the following outcomes in the 'Summary of findings' tables:

- proportion of participants developing a new pressure ulcer;

- time to complete ulcer development where analysed using appropriate survival analysis method;

- participant health-related quality of life/health status;

- participant comfort;

- cost effectiveness.

For review outcomes reported for comparisons not listed above, we will present a GRADE assessment without a 'Summary of findings' table.

When evaluating the 'Risk of bias' domain, we will downgrade the GRADE assessment only when we classified a study as being at high risk of bias for one or more domains, or when the 'Risk of bias' assessment for selection bias is unclear (i.e. classified as unclear for either the generation of the randomisation sequence or the allocation concealment domain). We will not downgrade for unclear 'Risk of bias' assessments in other domains.

We will select an informal optimal information size of 300 for binary outcomes, following the GRADE default value (Guyatt 2011). We will follow GRADE guidance and downgrade twice for imprecision when there are very few events and Cls around effects included both appreciable benefit and appreciable harm (considered GRADE 'default' of below 0.75 and above 1.25).

For calculating absolute risk differences for dichotomous and timeto-event outcomes, we will use the median of the risks in the control groups at particular time points.

\section{Subgroup analysis and investigation of heterogeneity}

Whilst we anticipate conducting an analysis of pressure redistributing chairs compared with standard chairs, we will assess potential heterogeneity across the following areas; specifically the type of pressure redistribution chair being used. Where there is evidence of between-trial heterogeneity, we envisage that we will conduct a subgroup analysis by chair type.

Where possible we will also present a subgroup analysis of trials which will include incidence of all grade of ulcers compared with trials which only measured ulcers of grade 2 and above.

Where possible we will perform subgroup analyses of the overall risk of bias category of a study (a binary comparison of: studies at low or unclear risk of bias compared with studies classed at high risk of bias).

\section{Sensitivity analysis}

Where possible we plan to perform sensitivity analyses to explore the effect of the following criteria:

- use of a fixed-effect versus a random-effects model;

- impact of missing data: our base case analysis will assume that participants with missing data did not develop new pressure ulcers. In this sensitivity analysis we will explore the impact of this assumption undertaking the analysis with complete case data only.

We have based elements of this Methods section on the standard Cochrane Wounds protocol template.

\section{ACK N O WLEDGEMENTS}

The review authors thank peer reviewers Chunhu Shi, Sharon Van Wicklin, Janet Wale and Emma Connaughton for their comments. They also thank Deirdre Walshe, who copy edited the protocol. 


\section{R E F E R E N C E S}

\section{Additional references}

\section{Bartley 2017}

Bartley C, Stephens M. Evaluating the impact of WaterCell ${ }^{\circledR}$ technology on pressure redistribution and comfort/discomfort of adults with limited mobility. Journal of Tissue Viability 2017; 26(2):144-9.

\section{Bhattacharya 2015}

Bhattacharya S, Mishra RK. Current understanding and newer modalities of treatment. Indian Journal of Plastic Surgery: Official Publication of the Association of Plastic Surgeons of India 2015; 48(1):4-16.

\section{Brazier 2002}

Brazier J, Roberts J, Deverill M. The estimation of a preferencebased measure of health from the SF-36. Journal of Health Economics 2002; 21(2):271-92.

\section{Cullum 2016}

Cullum N, Buckley H, Dumville J, Hall J, Lamb K, Madden M, et al. Wounds Research for Patient Benefit: A 5-Year Programme of Research. Programme Grants for Applied Research, No. 4.13. Southampton (UK): NIHR Journals Library, 2016.

\section{Deeks 2011}

Deeks JJ, Higgins JP, Altman DG. Chapter 9: Analysing data and undertaking meta-analyses. In: Higgins JP, Green S, editor(s). Cochrane Handbook for Systematic Reviews of Interventions Version 5.1.0 (updated March 2011). The Cochrane Collaboration, 2011. Available from handbook.cochrane.org.

\section{Demarré 2015}

Demarré L, Van Lancker A, Van Hecke A, Verhaeghe S, Grypdonck M, Lemey J, et al. The cost of prevention and treatment of pressure ulcers: a systematic review. International Journal of Nursing Studies 2015; 52(11):1754-74.

\section{EPUAP 2014}

European Pressure Ulcer Advisory Panel, National Pressure Ulcer Advisory Panel, Pan Pacific Pressure Injury Alliance. Prevention and treatment of pressure ulcers: quick reference guide. Emily Haesler (Editor). Cambridge Media: Osborne Park, Australia; 2014. www.epuap.org/wp-content/uploads/2016/10/ quick-reference-guide-digital-npuap-epuap-pppia-jan2016.pdf (accessed 17 March 2020).

\section{Gefen 2008}

Gefen A. How much time does it take to get a pressure ulcer? Integrated evidence from human, animal, and in vitro studies. Ostomy Wound Management 2008; 54(10):26-8, 30-5.

\section{Glanville 2019}

Glanville J, Dooley G, Wisniewski S, Foxlee R, Noel-Storr A. Development of a search filter to identify reports of controlled clinical trials within CINAHL Plus. Health Information \& Libraries Journal 2019; 36(1):73-90.

\section{Guest 2018}

Guest JF, Fuller GW, Vowden P, Vowden KR. Cohort study evaluating pressure ulcer management in clinical practice in the UK following initial presentation in the community: costs and outcomes. BMJ Open 2018; 8(7):e021769.

\section{Guyatt 2011}

Guyatt G, Oxman AD, Akl EA, Kunz R, Vist G, Brozek J, et al. GRADE guidelines: 1. Introduction-GRADE evidence profiles and summary of findings tables. Journal of Clinical Epidemiology 2011; 64(4):383-94.

\section{Harrand 2016}

Harrand J, Bannigan K. Do tilt-in-space wheelchairs increase occupational engagement: a critical literature review. Disability and Rehabilitation: Assistive Technology 2016; 11(1):3-12.

\section{Herdman 2011}

Herdman M, Gudex C, Lloyd A, Janssen M, Kind P, Parkin D, et al. Development and preliminary testing of the new fivelevel version of EQ-5D (EQ-5D-5L). Quality of Life Research: An International Journal of Quality of Life Aspects of Treatment, Care and Rehabilitation 2011; 20(10):1727-36. [PMID: 21479777]

\section{Higgins 2003}

Higgins JP, Thompson SG, Deeks JJ, Altman DG. Measuring inconsistency in meta-analyses. BMJ 2003; 327(7414):557-60.

\section{Higgins 2011a}

Higgins JP, Altman DG, Sterne JA. Chapter 8: Assessing risk of bias in included studies. In: Higgins JP, Green S, editor(s), Cochrane Handbook for Systematic Reviews of Interventions Version 5.1.0 (updated March 2011). The Cochrane Collaboration, 2011. Available from handbook.cochrane.org.

\section{Higgins 2011b}

Higgins JP, Deeks JJ, Altman DG. Chapter 16: Special topics in statistics. In: Higgins JP, Green S, editor(s), Cochrane Handbook for Systematic Reviews of Interventions Version 5.1.0 (updated March 2011). The Cochrane Collaboration, 2011. Available from handbook.cochrane.org.

\section{Jan 2010}

Jan YK, Jones MA, Rabadi MH, Foreman RD, Thiessen A. Effect of wheelchair tilt-in-space and recline angles on skin perfusion over the ischial tuberosity in people with spinal cord injury. Archives of Physical Medicine and Rehabilitation 2010; 91(11):1758-64.

\section{Jan 2013}

Jan YK, Crane BA, Liao F, Woods JA, Ennis WJ. Comparison of muscle and skin perfusion over the ischial tuberosities in response to wheelchair tilt-in-space and recline angles in people with spinal cord injury. Archives of Physical Medicine and Rehabilitation 2013; 94(10):1990-6.

\section{Kosiak 1959}

Kosiak M. Etiology and pathology of ischemic ulcers. Archives of Physical Medicine and Rehabilitation 1959; 40(2):62-9. 


\section{Krouskop 1983}

Krouskop TA. A synthesis of the factors that contribute to pressure sore formation. Medical Hypotheses 1983; 11(2):255-67.

\section{Lefebvre 2019}

Lefebvre C, Glanville J, Briscoe S, Littlewood A, Marshall C, , et al. Chapter 4: Searching for and selecting studies. In:. Higgins JPT, Thomas J, Chandler J, Cumpston M, Li T, Page MJ, Welch VA (editors) Cochrane Handbook for Systematic Reviews of Interventions version 6.0 (updated July 2019). Cochrane. Available from www.training.cochrane.org/handbook 2019.

\section{Liberati 2009}

Liberati A, Altman DG, Tetzlaff J, Mulrow C, Gotzsche PC, loannidis JP, et al. The PRISMA statement for reporting systematic reviews and meta-analyses of studies that evaluate health care interventions: explanation and elaboration. PLOS Medicine 2009; 6(7):e1000100.

\section{McInnes 2015}

McInnes E, Jammali-Blasi A, Bell-Syer SEM, Dumville JC, Middleton V, Cullum N. Support surfaces for pressure ulcer prevention. Cochrane Database of Systematic Reviews 2015, Issue 9. [DOI: 10.1002/14651858.CD001735.pub5]

\section{Michael 2007}

Michael SM, Porter D, Pountney TE. Tilted seat position for nonambulant individuals with neurological and neuromuscular impairment: a systematic review. Clinical Rehabilitation 2007; 21(12):1063-74.

\section{NHS Supply Chain 2019}

NHS Supply Chain. Medical furniture equipment, accessories, sackholders and bins. 2019. www.supplychain.nhs.uk/ product-information/contract-launch-brief/medical-furnitureequipment-accessories-and-sackholders/ (accessed 17 March 2020).

\section{NICE 2014}

National Institute of Health and Care Excellence. Pressure ulcers: prevention and management. Clinical guideline [CG179]. Published date: April 2014. www.nice.org.uk/guidance/cg179/ chapter/1-recommendations (accessed 17 March 2020).

\section{NPIAP 2016}

National Pressure Injury Advisory Panel. Pressure injury stages. 2016. npiap.com/general/custom.asp? page=PressurelnjuryStages (accessed 17 March 2020).

\section{NPIAP 2019}

National Pressure Injury Advisory Panel. Terms and definitions related to support surfaces. Revised 2019. npiap.com/page/ S3ITermsDefinitions (accessed 17 March 2020).

\section{Parmar 1998}

Parmar MK, Torri V, Stewart L. Extracting summary statistics to perform meta-analysis of the published literature for survival endpoints. Statistics in Medicine 1998; 17(24):2815-34.

\section{Reddy 2006}

Reddy M, Gill SS, Rochon PA. Preventing pressure ulcers: a systematic review. JAMA 2006; 296(8):974-84

\section{RESNA 2009}

RESNA Position on the application of tilt, recline, and elevating leg rests for wheelchairs. 2009. Assistive Technology (accessed 17 March 2020);21(1):13-22.

\section{Review Manager 2014 [Computer program]}

Nordic Cochrane Centre, The Cochrane Collaboration Review Manager 5 (RevMan 5). Version 5.3. Copenhagen: Nordic Cochrane Centre, The Cochrane Collaboration, 2014.

\section{Schubert 1994}

Schubert V, Héraud J. The effects of pressure and shear on skin microcirculation in elderly stroke patients lying in supine or semi-recumbent positions. Age and Aging 1994; 23(5):405-10.

\section{Schünemann 2011a}

Schünemann HJ, Oxman AD, Higgins JP, Vist GE, Glasziou P, Guyatt GH. Chapter 11: Presenting results and 'Summary of findings' tables. In: Higgins JP, Green S, editor(s), Cochrane Handbook for Systematic Reviews of Interventions Version 5.1.0 (updated March 2011). The Cochrane Collaboration, 2011. Available from handbook.cochrane.org.

\section{Schünemann 2011b}

Schünemann HJ, Oxman AD, Higgins JP, Deeks JJ, Glasziou P, Guyatt GH. Chapter 12: Interpreting results and drawing conclusions. In: Higgins JP, Green S, editor(s), Cochrane Handbook for Systematic Reviews of Interventions Version 5.1.0 (updated March 2011). The Cochrane Collaboration, 2011. Available from handbook.cochrane.org.

\section{Stephens 2017}

Stephens M, Bartley CA. Understanding the association between pressure ulcers and sitting in adults what does it mean for me and my carers? Seating guidelines for people, carers and health \& social care professionals. Journal of Tissue Viability 2017; 27(1):59-73.

\section{Sterne 2011}

Sterne JA, Egger M, Moher D. Chapter 10: Addressing reporting biases. In: Higgins JP, Green S, editor(s). Cochrane Handbook for Systematic Reviews of Interventions Version 5.1.0 (updated March 2011). The Cochrane Collaboration, 2011. Available from handbook.cochrane.org.

\section{Stevenson 2013}

Stevenson R, Collinson M, Henderson V, Wilson L, Dealey C, McGinnis $\mathrm{E}$, et al. The prevalence of pressure ulcers in community settings: an observational study. International Journal of Nursing Studies 2013; 50(11):1550-7.

\section{Stinson 2003}

Stinson MD, Porter-Armstrong A, Eakin P. Seat-interface pressure: a pilot study of the relationship to gender, body mass index, and seating position. Archives of Physical Medicine and Rehabilitation 2003; 84(3):405-9. 


\section{Thompson 1999}

Thompson SG, Sharp SJ. Explaining heterogeneity in metaanalysis: a comparison of methods. Statistics in Medicine 1999; 18(20):2693-708.

\section{Ware 1992}

, Sherbourne CD. The MOS 36-item short-form health survey (SF-36). I. Conceptual framework and item selection. Medical Care 1992; 30(6):473-83. [PMID: 1593914]

\section{Ware 1996}

, Kosinski M, Keller SD. A 12-item short-form health survey: construct of scales and preliminary tests of reliability and validity. Medical Care 1996; 34(3):220-33.

\section{Wheelchair Foundation 2019}

Wheelchair Foundation. Wheelchair needs in the world. www.wheelchairfoundation.org/about/ (accessed 17 March 2020).

\section{WHO 2018}

World Health Organization. International Classification of Diseases 11th Revision. 2018. icd.who.int/en (accessed 17 March 2020).

\section{AP PEN DICES}

\section{Appendix 1. Risk of bias assessment (individually randomised controlled trials)}

\section{Was the allocation sequence randomly generated?}

\section{Low risk of bias}

The investigators describe a random component in the sequence generation process such as: referring to a random number table; using a computer random number generator; coin tossing; shuffling cards or envelopes; throwing dice; drawing of lots.

\section{High risk of bias}

The investigators describe a non-random component in the sequence generation process. Usually, the description would involve some systematic, non-random approach, for example: sequence generated by odd or even date of birth; sequence generated by some rule based on date (or day) of admission; sequence generated by some rule based on hospital or clinic record number.

\section{Unclear}

Insufficient information about the sequence generation process provided to permit a judgement of low or high risk of bias.

\section{Was the treatment allocation adequately concealed?}

\section{Low risk of bias}

Participants and investigators enrolling participants could not foresee assignment because one of the following, or an equivalent method, was used to conceal allocation: central allocation (including telephone, internet-based, and pharmacy-controlled randomisation); sequentially-numbered drug containers of identical appearance; sequentially-numbered, opaque, sealed envelopes.

\section{High risk of bias}

Participants or investigators enrolling participants could possibly foresee assignments and thus introduce selection bias, such as allocation based on: use of an open random allocation schedule (e.g. a list of random numbers); assignment envelopes without appropriate safeguards (e.g. envelopes were unsealed, non-opaque, or not sequentially numbered); alternation or rotation; date of birth; case record number; any other explicitly unconcealed procedure.

\section{Unclear}

Insufficient information provided to permit a judgement of low or high risk of bias. This is usually the case if the method of concealment is not described, or not described in sufficient detail to allow a definite judgement, for example if the use of assignment envelopes is described, but it remains unclear whether envelopes were sequentially numbered, opaque, and sealed.

\section{Blinding - was knowledge of the allocated interventions adequately prevented during the study?}

\section{Low risk of bias}

Any one of the following:

- No blinding, but the review authors judge that the outcome and the outcome measurement are not likely to be influenced by lack of blinding.

- Blinding of participants and key study personnel ensured, and unlikely that the blinding could have been broken.

- Either participants or some key study personnel were not blinded, but outcome assessment was blinded and the non-blinding of others unlikely to introduce bias. 


\section{High risk of bias}

Any one of the following:

- No blinding or incomplete blinding, and the outcome or outcome measurement is likely to be influenced by lack of blinding.

- Blinding of key study participants and personnel attempted, but likely that the blinding could have been broken.

- Either participants or some key study personnel were not blinded, and the non-blinding of others likely to introduce bias.

\section{Unclear}

Either of the following:

- Insufficient information to permit judgement of low or high risk of bias.

- The study did not address this outcome.

\section{Were incomplete outcome data adequately addressed?}

\section{Low risk of bias}

Any one of the following:

- No missing outcome data.

- Reasons for missing outcome data are unlikely to be related to true outcome (for survival data, censoring unlikely to be introducing bias).

- Missing outcome data are balanced in numbers across intervention groups, with similar reasons for missing data across groups.

- For dichotomous outcome data, the proportion of missing outcomes compared with the observed event risk is not enough to have a clinically relevant impact on the intervention effect estimate.

- For continuous outcome data, a plausible effect size (difference in means or standardised difference in means) among missing outcomes is not enough to have a clinically relevant impact on the observed effect size.

- Missing data have been imputed using appropriate methods.

\section{High risk of bias}

Any one of the following:

- Reason for missing outcome data are likely to be related to the true outcome, with either an imbalance in numbers or reasons for missing data across intervention groups.

- For dichotomous outcome data, the proportion of missing outcomes compared with the observed event risk is enough to induce clinically relevant bias in the intervention effect estimate.

- For continuous outcome data, a plausible effect size (difference in means or standardised difference in means) among missing outcomes is enough to induce a clinically relevant bias in the observed effect size.

- 'As-treated' analysis done with a substantial departure of the intervention received from that assigned at randomisation.

- Potentially inappropriate application of simple imputation.

\section{Unclear}

Either of the following:

- Insufficient reporting of attrition/exclusions to permit a judgement of low or high risk of bias (e.g. number randomised not stated, no reasons for missing data provided).

- The study did not address this outcome.

\section{Are reports of the study free of suggestion of selective outcome reporting?}

\section{Low risk of bias}

Either of the following:

- The study protocol is available and all of the study's prespecified (primary and secondary) outcomes that are of interest in the review have been reported in the prespecified way.

- The study protocol is not available but it is clear that the published reports include all expected outcomes, including those that were prespecified (convincing text of this nature may be uncommon).

\section{High risk of bias}

Any one of the following: 
- Not all of the study's prespecified primary outcomes have been reported.

- One or more primary outcomes is/are reported using measurements, analysis methods, or subsets of the data (e.g. subscales) that were not prespecified.

- One or more reported primary outcomes was/were not prespecified (unless clear justification for their reporting is provided, such as an unexpected adverse effect).

- One or more outcomes of interest in the review is/are reported incompletely so that they cannot be entered in a meta-analysis.

- The study report fails to include results for a key outcome that would be expected to have been reported for such a study.

\section{Unclear}

Insufficient information provided to permit a judgement of low or high risk of bias. It is likely that most studies will fall into this category.

\section{Other sources of potential bias}

\section{Low risk of bias}

The study appears to be free of other sources of bias.

\section{High risk of bias}

There is at least one important risk of bias. For example, the study:

- had a potential source of bias related to the specific study design used; or

- has been claimed to have been fraudulent; or

- had some other problem.

\section{Unclear}

There may be a risk of bias, but there is either:

- insufficient information to assess whether an important risk of bias exists; or

- insufficient rationale or evidence that an identified problem will introduce bias.

\section{Appendix 2. Risk of bias (cluster-randomised controlled trials)}

In cluster-randomised trials, particular biases to consider include: (i) recruitment bias; (ii) baseline imbalance; (iii) loss of clusters; (iv) incorrect analysis; and (v) comparability with individually randomised trials.

(i) Recruitment bias can occur when individuals are recruited to the trial after the clusters have been randomised, as the knowledge of whether each cluster is an 'intervention' or 'control' cluster could affect the types of participants recruited.

(ii) Cluster-randomised trials often randomise all clusters at once, so lack of concealment of an allocation sequence should not usually be an issue. However, because small numbers of clusters are randomised, there is a possibility of chance baseline imbalance between the randomised groups, in terms of either the clusters or the individuals. Although not a form of bias as such, the risk of baseline differences can be reduced by using stratified or pair-matched randomisation of clusters. Reporting of the baseline comparability of clusters, or statistical adjustment for baseline characteristics, can help reduce concern about the effects of baseline imbalance.

(iii) Occasionally complete clusters are lost from a trial, and have to be omitted from the analysis. Just as for missing outcome data in individually randomised trials, this may lead to bias. In addition, missing outcomes for individuals within clusters may also lead to a risk of bias in cluster randomised trials.

(iv) Many cluster randomised trials are analysed by incorrect statistical methods, not taking the clustering into account. Such analyses create a 'unit of analysis error' and produce over-precise results (the standard error of the estimated intervention effect is too small) and $P$ values that are too small. They do not lead to biased estimates of effect. However, if they remain uncorrected, they will receive too much weight in a meta-analysis.

(v) In a meta-analysis including both cluster and individually randomised trials, or including cluster-randomised trials with different types of clusters, possible differences between the intervention effects being estimated need to be considered. For example, in a vaccine trial of infectious diseases, a vaccine applied to all individuals in a community would be expected to be more effective than if the vaccine was applied to only half of the people. Another example is provided by a Cochrane Review of hip protectors (Hahn 2005). The cluster trials showed large positive effect whereas individually randomised trials did not show any clear benefit. One possibility is that there was a 'herd effect' in the cluster-randomised trials (which were often performed in nursing homes, where compliance with using the protectors may have been enhanced). In general, such 'contamination' would lead to underestimates of effect. Thus, if an intervention effect is still demonstrated despite contamination in those trials that were not cluster randomised, a confident conclusion about the presence of an effect can be drawn. However, the size of the effect is likely to be underestimated. Contamination and 'herd effects' may be different for different types of cluster. 


\section{Appendix 3. Cochrane Central Register of Controlled Trials (CENTRAL) draft search strategy}

\#1 MeSH descriptor: [Pressure Ulcer] explode all trees

\#2 (pressure next (ulcer ${ }^{\star}$ or sore ${ }^{\star}$ or injur $\left.{ }^{\star}\right)$ ):ti,ab,kw

\#3 (decubitus next (ulcer ${ }^{\star}$ or sore $\left.{ }^{\star}\right)$ ):ti,ab,kw

\#4 (bedsore ${ }^{\star}$ or bed sore ${ }^{\star}$ ):ti,ab,kw

\#5 \#1 or \#2 or \#3 or \#4

\#6 MeSH descriptor: [Wheelchairs] explode all trees

\#7 MeSH descriptor: [Posture] explode all trees

\#8 MeSH descriptor: [Ergonomics] explode all trees

\#9 (wheelchair* or wheel-chair ${ }^{\star}$ or chair ${ }^{\star}$ or armchair ${ }^{\star}$ or arm-chair ${ }^{\star}$ or seat or seats or seated or seating or sit or sitting):ti,ab,kw

\#10 (riser-recline ${ }^{\star}$ or (riser recline $\left.{ }^{\star}\right)$ ):ti,ab,kw

\#11 (tilt-in-space):ti,ab,kw

\#12 (backrest* or back-rest* or armrest* or arm-rest* or legrest* or leg-rest $\left.{ }^{\star}\right): t i, a b, k w$

$\# 13 \# 6$ or \#7 or \#8 or \#9 or \#10 or \#11 or \#12

\#14 \#5 and \#13

\section{H ISTORY}

Protocol first published: Issue 6, 2020

\section{CONTRIBUTIONSOFAUTHORS}

Melanie Stephens: conceived the review question; developed the protocol; coordinated the protocol development; produced the first draft of the protocol; contributed to writing and editing the protocol; advised on the protocol; and approved the final version of the protocol prior to submission.

Carol Bartley: conceived the review question; developed the protocol; coordinated the protocol development; produced the first draft of the protocol; contributed to writing and editing the protocol; advised on the protocol; and approved the final version of the protocol prior to submission.

Jo Dumville: contributed to writing and editing the protocol; advised on the protocol; and approved the final version of the protocol prior to submission.

Christopher Cammiss: contributed to editing the protocol; advised on the protocol; and approved the final version of the protocol prior to submission.

\section{Contributions of the Editorial Base}

Nicky Cullum (Joint Co-ordinating Editor): edited the protocol; advised on methodology, interpretation and content and approved the final version of the protocol prior to submission.

Gill Rizzello (Managing Editor): coordinated the editorial process; advised on content; and edited the protocol.

Sophie Bishop (Information Specialist): designed the search strategy and edited the search methods section.

Tom Patterson (Editorial Assistant): edited the reference sections.

\section{DECLARATIONS OF INTEREST}

Melanie Stephens: the University of Salford received funding from CareFlex to conduct a small pilot study to test the pressure redistributing properties of three chairs manufactured by this company. This also included funding to present the findings at the OT Show. I had no control over the use of these funds. 
Carol Bartley: the University of Salford received funding from CareFlex to conduct a small pilot study to test the pressure redistributing properties of three chairs manufactured by this company. This also included funding to present the findings at the OT Show. I had no control over the use of these funds.

Jo Dumville: received research funding from the National Institute for Health Research (NIHR) for the production of systematic reviews focusing on high-priority Cochrane Reviews in the prevention and treatment of wounds. This research was co-funded by the NIHR Manchester Biomedical Research Centre, and partly funded by the NIHR Collaboration for Leadership in Applied Health Research and Care (CLAHRC) Greater Manchester.

Christopher Cammis: none known.

\section{SOURCES OF SUPPORT}

\section{Internal sources}

- Division of Nursing, Midwifery and Social Work, School of Health Sciences, Faculty of Biology, Medicine and Health, University of Manchester, UK

\section{External sources}

- National Institute for Health Research Collaboration for Leadership in Applied Health Research and Care (NIHR CLAHRC) Greater Manchester Centre, UK

Jo Dumville was partly funded by the NIHR CLAHRC Greater Manchester Centre. The funder had no role in the decision to publish, or preparation of the manuscript. However, the review may be considered to be affiliated to the work of the NIHR CLAHRC Greater Manchester Centre. The views expressed herein are those of the review authors and not necessarily those of the NHS, the NIHR, or the Department of Health and Social Care.

- NIHR Manchester Biomedical Research Centre, UK

This review was co-funded by the NIHR Manchester Biomedical Research Centre. The views expressed in this publication are those of the author(s) and not necessarily those of the NHS, the NIHR, or the Department of Health and Social Care.

- National Institute for Health Research (NIHR), UK

This project was supported by the National Institute for Health Research, via Cochrane Infrastructure funding to Cochrane Wounds. The views expressed are those of the authors and not necessarily those of the NIHR or the Department of Health and Social Care. 